\title{
Fratura em mandíbula atrófica: relato de caso
}

\author{
Atrophic mandible fracture: case report \\ Fractura atrófica en mandíbula: reporte de caso
}

\author{
Henrique Celestino LIMA E SILVA ${ }^{\mathbf{1}}$ \\ Larissa Reale KNOLL ${ }^{2}$ \\ Sérgio Luís de MIRANDA ${ }^{3}$ \\ Roberto MORENO4 \\ ${ }^{1}$ Especialista em Cirurgia e Traumatologia Bucomaxilofacial (CTBMF); \\ Mestrando do Programa de Pós-Graduação em Odontologia da Universidade Federal de Mato Grosso do Sul-UFMS,
Campo Grande-MS, Brasil \\ ${ }^{2}$ Especialista em Ortodontia pela SPO-SP \\ ${ }^{3}$ Especialista em Cirurgia e Traumatologia Bucomaxilofacial (CTBMF); \\ Especialista em Otorrinolaringologia, Cirurgia de Cabeça e Pescoço; \\ Mestre e Doutor em Otorrinolaringologia e Cirurgia de Cabeça e Pescoço pela Universidade Federal de São Paulo. \\ UNIFESP, São Paulo-SP, Brasil; \\ Cirurgião Craniomaxilofacial do Hospital Israelita Albert Einstein, São Paulo-SP, Brasil \\ ${ }^{4}$ Especialista em Cirurgia e Traumatologia Bucomaxilofacial (CTBMF); \\ Mestre e Doutor em Otorrinolaringologia e Cirurgia de Cabeça e Pescoço pela Universidade Federal de São Paulo, \\ UNIFESP, São Paulo-SP, Brasil; \\ Cirurgião Craniomaxilofacial do Hospital Israelita Albert Einstein, São Paulo-SP, Brasil
}

\begin{abstract}
Resumo
A atrofia da mandíbula, normalmente encontrada em pacientes idosos ou em virtude da perda dentária precoce, torna tal osso mais suscetível a fraturas e a qualidade do tecido, prejudicada por alterações fisiológicas, torna o reparo dessas fraturas mais complexo. As fraturas em mandíbulas atróficas representam um capítulo particular no trauma facial, pois as características das lesões nessas situações diferem daquelas que ocorrem em pacientes dentados, necessitando, muitas vezes, tratamentos mais complexos. O manejo de tais lesões deve levar em consideração a idade e a condição sistêmica do paciente, o tempo decorrido desde o trauma e a complexidade do dano. O objetivo do estudo foi descrever um caso cirúrgico de fratura de mandíbula atrófica, onde foi necessário duas abordagens cirúrgicas.

Descritores: Atrofia; Mandíbula; Reconstrução Mandibular.
\end{abstract}

\begin{abstract}
The mandibular atrophy, usually found in elderly patient or due to the early tooth loss, makes that bone more susceptible to fractures and the tissue quality, damaged by physiological changes, makes the repair of these fractures more complex. Fractures in atrophic mandibles represent a particular chapter of facial trauma, because the trauma features in these cases differ from those involving dentulous patients, necessitating, sometimes, more complex treatments. The management of such injuries should take into consideration the age and the systemic condition of the patient, the time since the trauma and the complexity of damage. The aim of the study was to describe a surgical case of atrophic mandible fracture, which was required treatment with two surgical approaches.
\end{abstract}

Descriptors: Atrophy; Mandible; Mandibular Reconstruction.

\section{Resumen}

La atrofia mandibular, que normalmente se encuentran en los pacientes de edad avanzada o debido a la pérdida prematura de dientes, hace que el hueso más susceptibles a las fracturas y la calidad del tejido, dañadas por cambios fisiológicos, hace que la reparación de estas fracturas más complejo. Las fracturas en mandíbulas atróficas representan un capítulo particular de un traumatismo facial, debido a las características de trauma en estos casos difieren de aquellas envolviendo pacientes dentados, lo que exige, a veces, la gestión tratamientos. El más complejo de este tipo de lesiones se debe tomar en consideración la edad y la condición sistémica de el paciente, el tiempo transcurrido desde el trauma y la complejidad de los daños. El objetivo del estudio fue describir un caso quirúrgico de la fractura de mandíbula atrófica, que se requiere dos enfoques quirúrgicos.

Descriptores: Atrofia; Mandíbula; Reconstrucción Mandibular.

\section{INTRODUÇÃO}

As fraturas em mandíbulas atróficas são mais comumente encontradas em pacientes idosos, devido à perda precoce dos elementos dentais. Nestes pacientes, as alterações fisiológicas e/ou anatômicas, inerentes da senilidade têm uma influência negativa sobre a reparação óssea. Fatores relacionados à atrofia mandibular, como a quantidade e qualidade ósseas, área reduzida de contato entre os segmentos fraturados e suprimento sanguíneo inadequado, além da alta incidência de alterações sistêmicas nestes pacientes, aumentam a complexidade do tratamento ${ }^{1}$.
Os principais agentes etiológicos das fraturas de mandíbula apresentados por esta população são os acidentes automobilísticos, seguidos dos episódios de quedas e as agressões, sendo as mulheres mais afetadas que os homens ${ }^{2}$. A classificação das fraturas mandibulares atróficas foi definida na literatura com base na altura do osso fraturado, dessa forma foi descrito que uma mandíbula inferior a 20 $\mathrm{mm}$ de altura em região de fratura deve ser classificada como "atrófica". A classificação foi dividida em Classe I com altura entre 20 e $16 \mathrm{~mm}$, Classe II de 15 a $11 \mathrm{~mm}$ e por 
fim Classe III com altura inferior a $10 \mathrm{~mm}^{3}$.

Para eleição da técnica cirúrgica e prognostico do tratamento o cirurgião deve ponderar a condição sistêmica do paciente ${ }^{4,5}$. Já em relação à técnica cirúrgica indica-se o acesso extraoral para as fraturas de mandíbulas atróficas severas, devido a necessidade de grande exposição cirúrgica e para satisfatória redução e fixação ${ }^{6}$.

O presente estudo tem como objetivo relatar um caso clínico cirúrgico de tratamento de fratura de mandíbula atrófica em paciente idoso, onde foi necessário duas abordagens cirúrgicas, a primeira devido o trauma inicial e a segunda devido a refratura após 6 anos do tratamento inicial.

\section{CASO CLÍNICO}

Paciente 80 anos de idade, sexo masculino procurou atendimento em hospital particular na cidade de São Paulo $\mathrm{SP}$, onde a equipe realizou atendimento inicial no setor de Cirurgia e Traumatologia Bucomaxilofacial, com relato de queda da própria altura, dor em face e dificuldade de mastigação. Ao exame clínico notou-se assimetria facial onde se observava edema moderado em hemiface esquerda com perda de proeminência mentoniana e equimose submentoniana. Ao exame intrabucal foi verificado hematoma sublingual, rebordos maxilares atróficos, descontinuidade óssea de arco mandibular, instabilidade oclusal e crepitação à palpação, notou-se também que o paciente fazia uso de prótese total superior e inferior. $\mathrm{Na}$ investigação diagnóstica, a tomografia computadorizada (TC) de face mostrou imagens de fraturas em mandíbula, notou-se fratura em região do corpo de mandíbula esquerda e fratura em região de para-sínfise direita (Figuras 1 a 3).

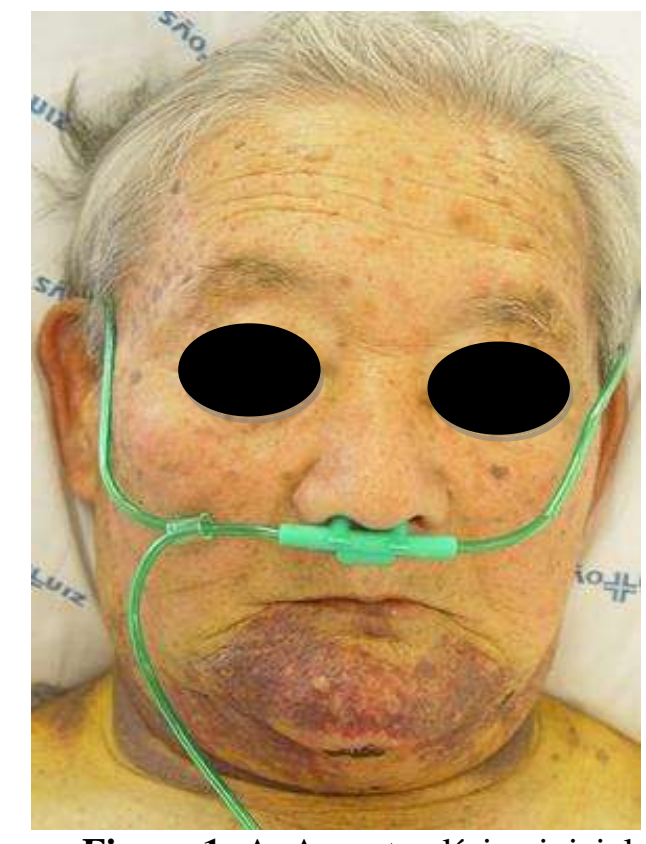

Figura 1: A. Aspecto clínico inicial.

A história médica familiar não mostrou nenhum dado significativo e o paciente não apresentava nenhuma patologia de base. Após a avaliação do risco cirurgico, o tratamento da fratura foi realizado por meio de intervenção cirúrgica em ambiente hospitalar, sob anestesia geral, e intubação nasotraqueal. Na sequência foi demarcada incisão em região submandibular direita, submentual e submandibular esquerda. $\mathrm{O}$ acesso realizado foi o acesso extraoral transcervical. Após o acesso da região foi realizada redução cirúrgica cruenta com simplificação da fratura por meio de placas e parafusos. Com cotos ósseos em posição seguiu-se o tratamento com a fixação interna rígida utilizando uma placa de reconstrução do sistema $2.4 \mathrm{~mm}$, dessa forma obtendo uma satisfatória fixação. A sutura foi realizada por planos (Figuras 4 e 5).

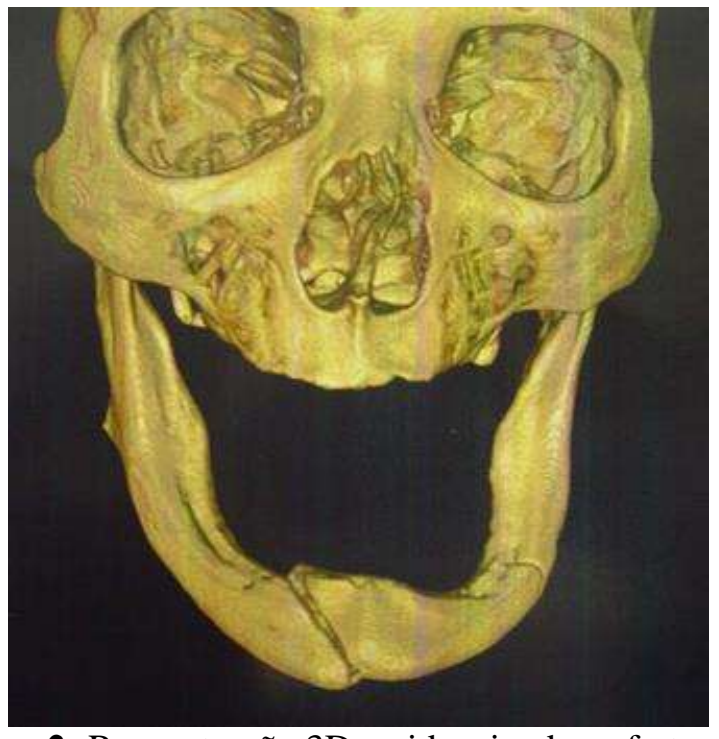

Figura 2: Reconstrução 3D, evidenciando as fraturas.

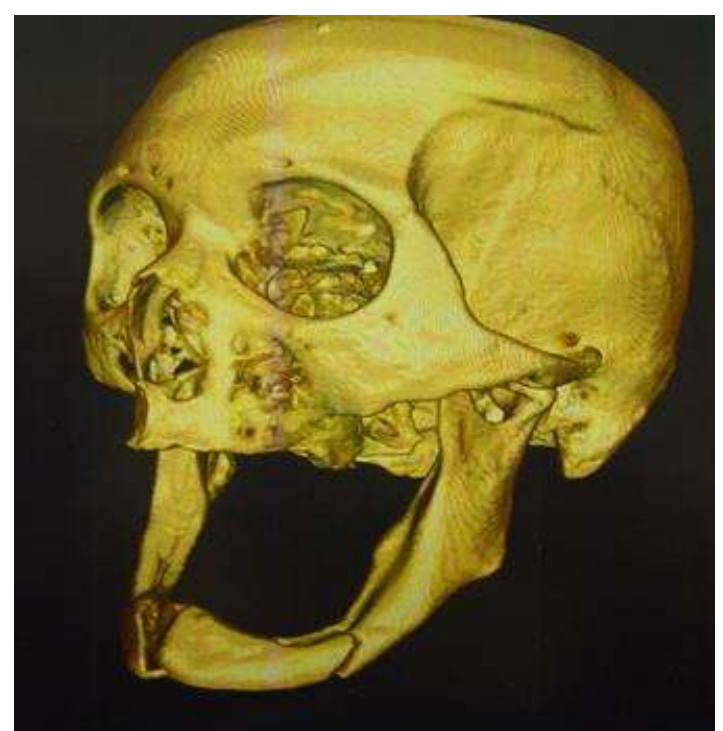

Figura 3: Reconstrução 3D, evidenciando as fraturas.

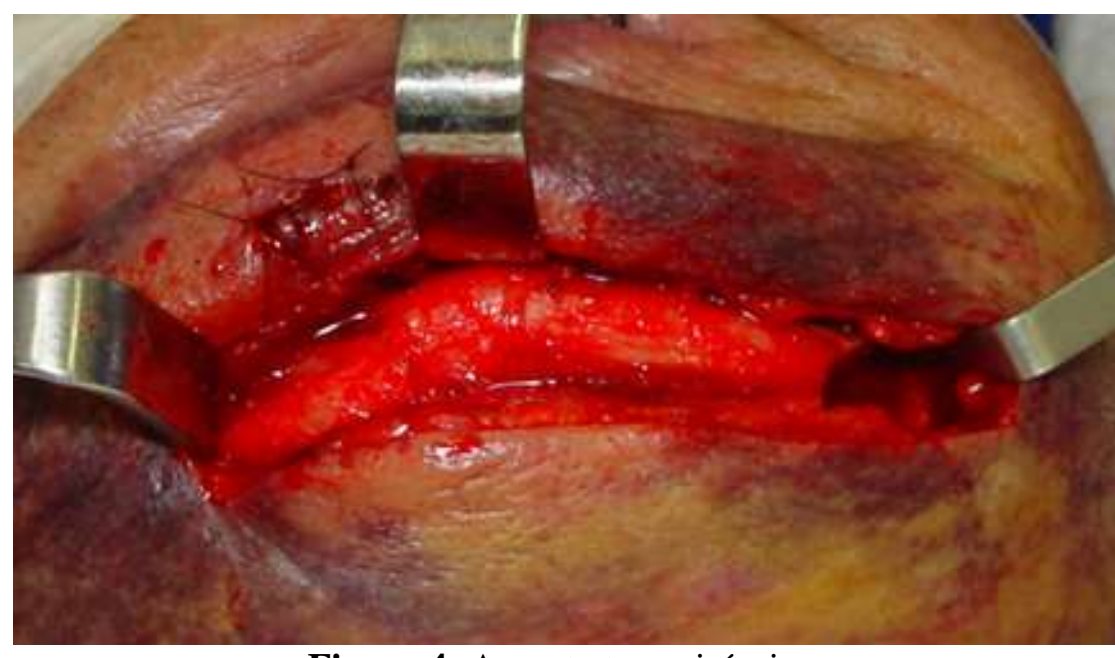

Figura 4: Aspecto transcirúrgico.

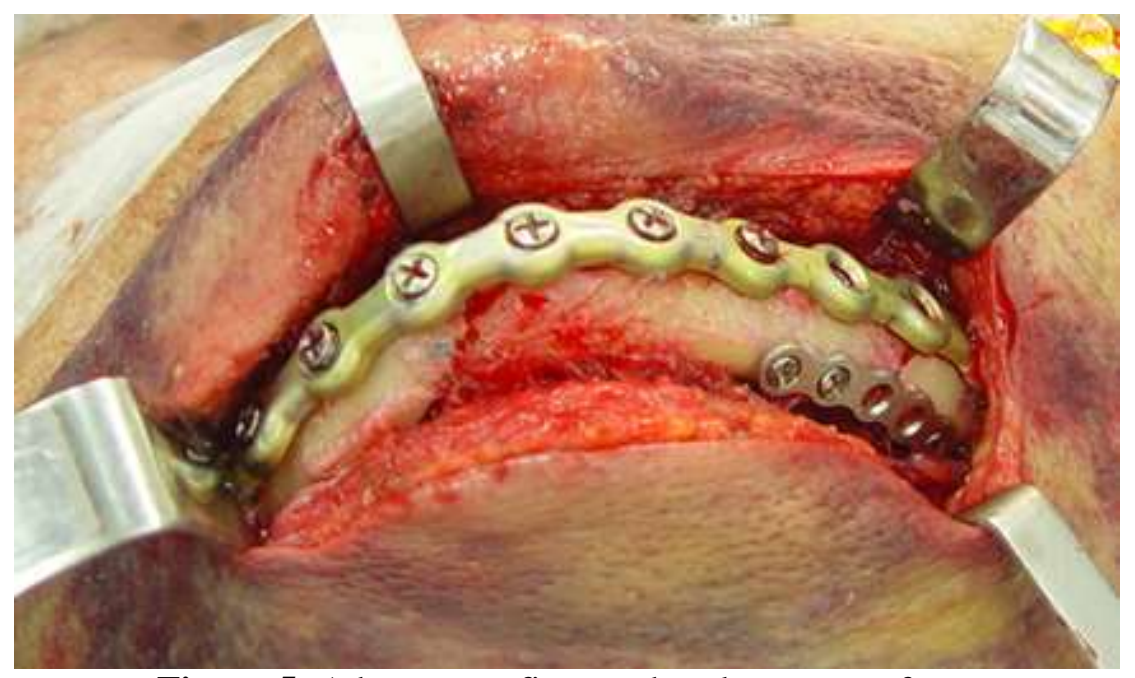

Figura 5: Adaptação e fixação das placas e parafusos

No pós-operatório de um mês o paciente apresentou boa abertura bucal, ausência de parestesia, manutenção da movimentação do lábio inferior e bom aspecto cicatricial, dessa forma obtendo um satisfatório aspecto estético e funcional para o paciente. Também foi solicitada radiografia panorâmica onde se observou ótima adaptação das placas e 
parafusos, obtendo alinhamento mandibular adequado (Figuras 6 a 8).

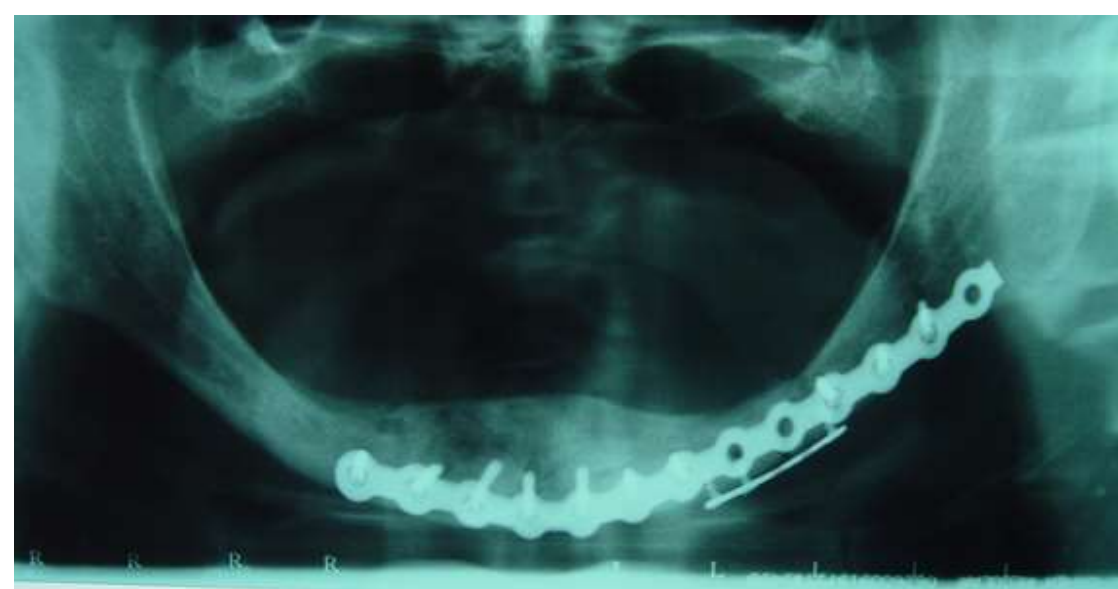

Figura 6: Pós-operatório de um mês. Radiografia panorâmica evidenciando uma ótima adaptação das placas e parafusos, obtendo alinhamento mandibular adequado.

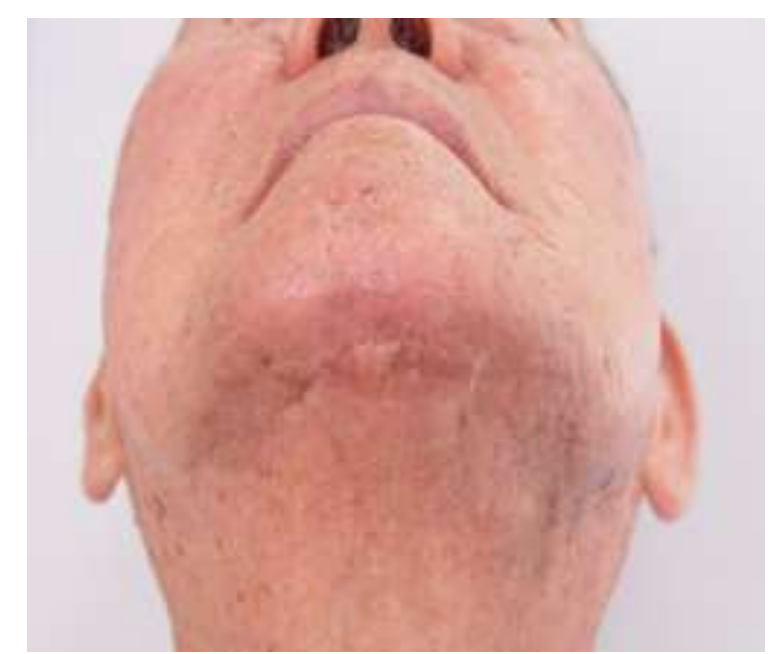

Figura 7: Aspecto clínico final com um satisfatório aspecto estético e funcional para o paciente.

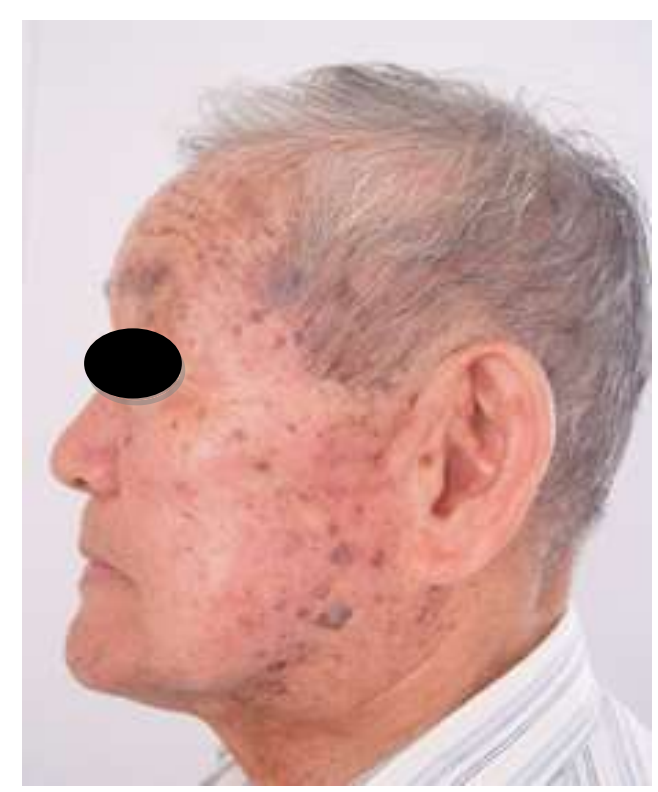

Figura 8: Aspecto clínico final com um satisfatório aspecto estético e funcional para o paciente.

Após seis anos de tratamento o paciente sofreu novamente uma queda da própria altura e evoluindo novamente com fraturas em mandíbula, porém desta vez uma fratura em corpo de mandíbula direita e outra em região de parasínfise esquerda (Figuras 9 a 11). O mesmo foi submetido a uma segunda intervenção cirúrgica para redução e fixação das fraturas. As placas e parafusos foram removidos e substituídos por um novo material de fixação, a simplificação da fratura foi por meio de placas e parafusos, os fragmentos foram estabilizados utilizando placa de reconstrução do sistema $2.4 \mathrm{~mm}$ (Figuras 12 e 13). No pósoperatório de seis meses pode se observar a adaptação de placas, parafusos e satisfatório resultado estético e funcional (Figura 14).

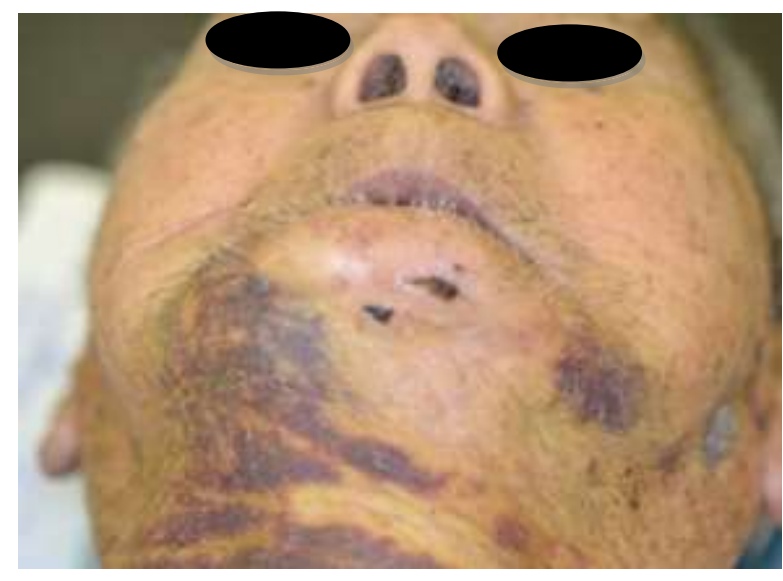

Figura 9: Aspecto clínico após o segundo trauma.

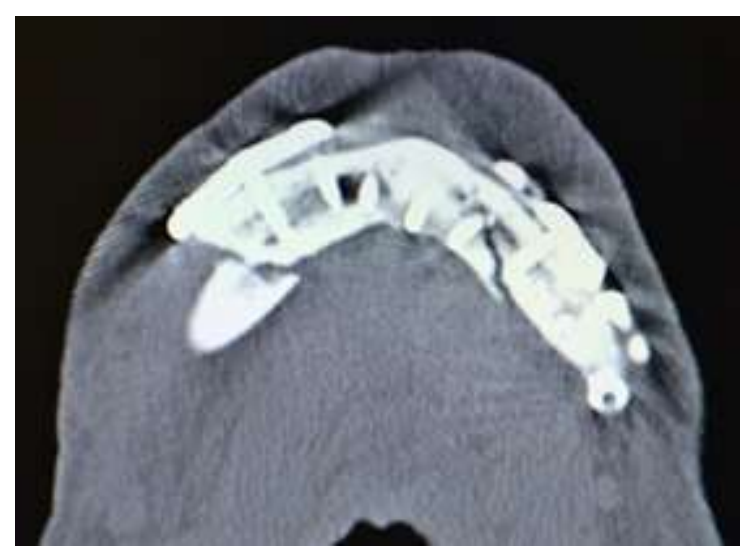

Figura 10: Tomografia computadorizada de face (TC), corte axial, evidenciando as fraturas.

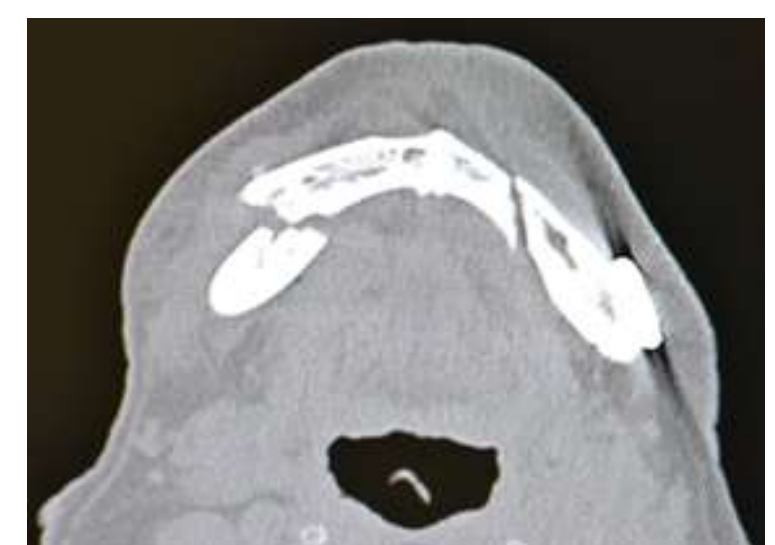

Figura 11: Tomografia computadorizada de face (TC), corte axial, evidenciando as fraturas.

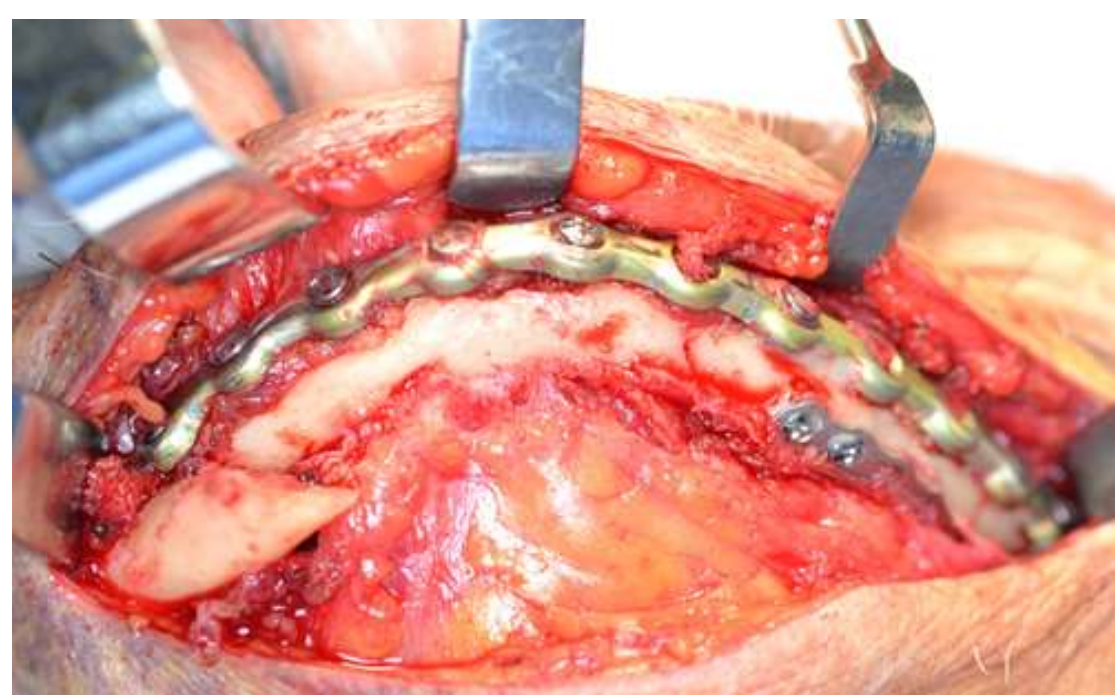

Figura 13: Aspecto transcirúrgico evidenciando traços e deslocamento das fraturas.

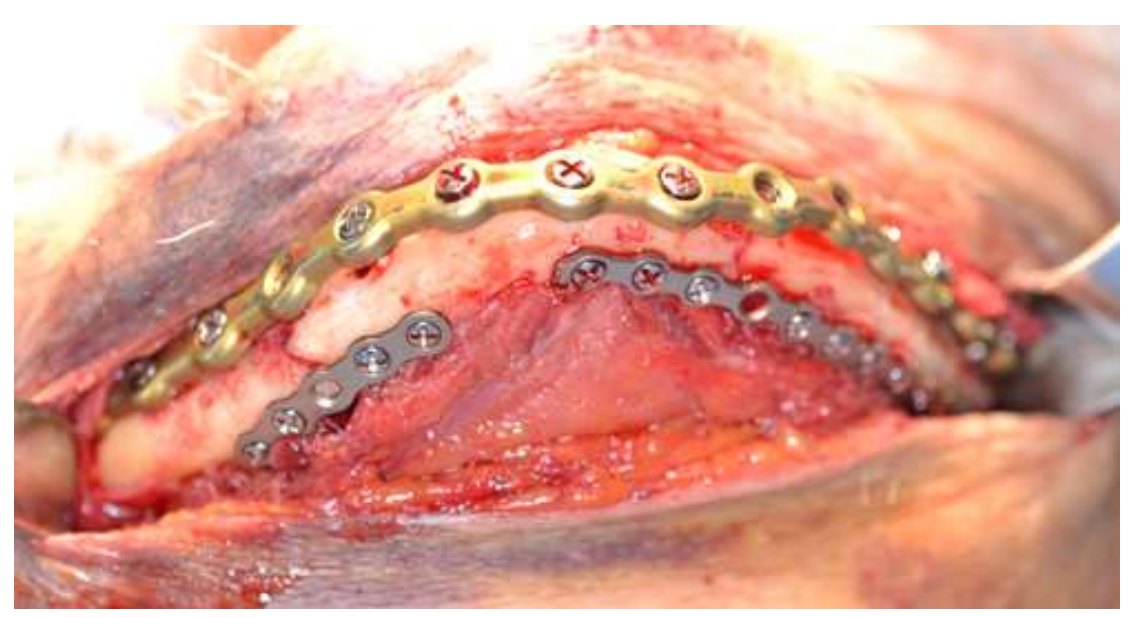

Figura 14: Redução e fixação das fraturas. 


\section{DISCUSSÃO}

O tratamento da fratura mandibular objetiva o restabelecimento da oclusão funcional e a continuidade mandibular. Diversas são as técnicas de fixação para essas fraturas. A contenção interna com fio de aço tem entrado em desuso por ter levado, no passado, a muitos casos de infecção, implicando em significante perda óssea e morbidade ${ }^{5}$. Além disso, o inconveniente de requerer, pelo menos, quatro semanas de restrição à movimentação mandibular, exigindo colaboração contínua por parte dos pacientes.

O tratamento conservador ainda gera resultados imprevisíveis, o que leva os profissionais a optarem pelo tratamento cirúrgico. Atualmente, as placas de titânio são amplamente utilizadas para a fixação interna estável, restabelecendo a união dos ossos fraturados e deslocados, dando-lhes estabilidade ${ }^{7}$.

Para compreender melhor, Prein $(1998)^{8}$ classifica a fixação interna rígida em dois tipos: 1) "Load Sharing" quando há boa quantidade de osso disponível, em que as cargas funcionais são divididas entre o osso remanescente e o sistema de fixação; 2) "Load Bearing", no qual o sistema de fixação suporta toda ou quase toda carga funcional, até que ocorra a reparação óssea, como é o caso das fraturas cominutivas em mandíbulas atróficas e dos enxertos ósseos livres segmentados, realizados na mandíbula. Desta forma, as mandíbulas atróficas necessitam de grande resistência da fixação, mesmo em pacientes com musculatura pobre e força muscular aparentemente reduzida. Ocorre que, com a diminuição da altura mandibular gerada pela atrofia, acarreta-se na alteração da disposição das zonas de tensão, neutra e compressão, as quais se desenvolvem na mandíbula, em decorrência da solicitação funcional, aproximando-as. Como resultado, uma única placa reconstrutiva terá que suportar toda carga funcional. O não conhecimento destes conceitos pode provocar complicações que incluem: infecções, não união, má união e fratura da placa ${ }^{9}$.

A osteossíntese devera ser mais forte, quanto mais atrófica for a mandíbula, dessa forma a força fisiológica aplicada em mandíbula se dissipa sobre o material de fixação "Load-Bearing", garantindo maior estabilidade e não havendo compartilhamento de carga com o osso ${ }^{10,11}$, fundamento utilizado no caso descrito.

Dois acessos cirúrgicos são mais citados na literatura, o acesso intra oral e o acesso extra oral, devido a dificuldade de redução, estabilização, extensão da fratura ${ }^{6,10}$ e para obter uma fixação adequada o caso deste estudo foi tratado com acesso extra oral.

Com o objetivo de minimizar as complicações pósoperatórias referentes a má nutrição sanguínea da mandíbula atrófica fraturada, é fundamental que o periósteo seja preservado, levanto em consideração que a própria fratura pode comprometer a vascularização mandibular e todo trauma ao osso irá produzir certa quantidade de dano $\operatorname{vascular}^{12}$.

\section{CONCLUSÃO}

A individualização do tratamento de fraturas mandibulares é de fundamental importância, especialmente ao lidar pacientes edêntulos que apresentam mandíbulas atróficas, pois não raramente, estes possuem uma condição sistêmica que dificulta a abordagem cirúrgica. O conhecimento da fixação interna rígida "Load Bearing" mostra-se como um excelente aliado, quando se pretende diminuir as complicações pós-operatórias e obter uma

satisfatória redução, estabilização de fraturas em mandíbulas atróficas severas.

\section{REFERÊNCIAS}

1. Marciani RD. Invasive management of the fractured atrophic edentulous mandible. J Oral Maxillofac Surg. 2001; 59(7):792-5.

2. Fonseca, RJ. Oral and Maxillofacial Trauma, 3 ed. Philadelphia: W.B. Saunders Company, v. 3, 2000, 500p.

3. Luhr HG, Reidick T, Merten HA. Results of treatment of fractures of the atrophic edentulous mandible by compression plating: aretrospective evaluation of 84 consecutive cases. J Oral Maxillofac Surg. 199654(3):250-4

4. Torriani MA, Oliveira MG. O Cirurgião Dentista, sua formação e sua prática no atendimento ao idoso portador de traumatismo bucomaxilofacial. Rev Odont Ciência. 2000; 31(3):116-23.

5. Ellis E 3rd, Price C. Treatment protocol for fractures of the atrophic mandible. J Oral Maxillofac Surg. 2008; 66(3):421-35.

6. Ehrenfeld M, Manson PN, Prein J. Principles of internal fixation of the craniomxillofacial skeleton: trauma and orthognathic surgery. Davos: AO Foundation; 2012. p.170-2.

7. Luhr HG, Reidick T, Merten HA. Results of treatment of fractures of the atrophic edentulous mandible by compression plating: a retrospective evaluation of 84 consecutive cases. J Oral Maxillofac Surg. 1996; 54(3): 250-4.

8. Prein J. Manual of internal fi xation in the cranio-facial skeleton. Berling Heidelberg: Springer-Verlag; 1998.

9. Spina AM, Marciani RD. Mandibular fractures. In: Fonseca RJ, Marciani RD, Hendler BH. Oral and maxillofacial surgery. vol 3. Philadelphia: Saunders; 2000. p.103-7.

10. Wittwer G, Adeyemo WL, Turhani D, Ploder O. Treatment of atrophic mandibular fractures based on the degree of atrophyexperience with different plating systems: a retrospective study. J Oral Maxillofac Surg. 2006; 64(2):230-4.

11. Bruce RA, Ellis E 3rd. The second Chalmers J. Lyons Academy study of fractures of the edentulous mandible. J Oral Maxillofac Surg. 1993; 51(8):904-11.

12. McCarthy I. The physiology of bone blood flow: a review. J Bone Joint Surg Am. 2006; 88(2):4-9.

\section{CONFLITO DE INTERESSES}

Os autores declaram não haver conflitos de interesse.

\section{AUTOR PARA CORRESPONDÊNCIA}

Henrique Celestino Lima e Silva

henriquelimacirurgia@gmail.com 